Article

\title{
Searching for Jet Emission in LMXBs: A Polarimetric View
}

\author{
Maria Cristina Baglio 1,2,* (1), Paolo D'Avanzo ${ }^{2}$, Sergio Campana ${ }^{2}$ (i) and Dave M. Russell ${ }^{1}$ \\ 1 Physics Department, New York University Abu Dhabi, PO Box 129188, Abu Dhabi, UAE; \\ dave.russell@nyu.edu \\ 2 INAF, Osservatorio Astronomico di Brera, via E. Bianchi 46, 23807 Merate, Italy; \\ paolo.davanzo@brera.inaf.it (P.D.A.); sergio.campana@brera.inaf.it (S.C.) \\ * Correspondence: mcb19@nyu.edu
}

Academic Editors: Emmanouil Angelakis, Markus Boettcher and Jose L. Gómez

Received: 25 August 2017; Accepted: 25 September 2017; Published: 7 October 2017

\begin{abstract}
We present results taken from a study aiming at detecting the emission from relativistic particles jets in neutron star-low mass X-ray binaries using optical polarimetric observations. First, we focus on a polarimetric study performed on the persistent LMXB 4U $0614+091$. Once corrected for interstellar effects, we measured an intrinsic linear polarization in the $r$-band of $\sim 3 \%$ at a $3 \sigma$ confidence level. This is in-line with the observation of an infrared excess in the spectral energy distribution (SED) of the source, reported in a previous work, which the authors linked to the optically thin synchrotron emission of a jet. We then present a study performed on the transitional millisecond pulsar PSR J1023+0038 during quiescence. We measured a linear polarization of $1.09 \pm 0.27 \%$ and $0.90 \pm 0.17 \%$ in the $V$ and $R$ bands, respectively. The phase-resolved polarimetric curve of the source in the $R$-band reveals a hint of a sinusoidal modulation at the source orbital period. The NIR -optical SED of the system did not suggest the presence of a jet. We conclude that the optical linear polarization observed for PSR J1023+0038 is possibly due to Thomson scattering with electrons in the disc, as also suggested by the hint of the modulation of the $R$-band linear polarization at the system orbital period.
\end{abstract}

Keywords: jets; polarization; accretion disc

\section{Introduction}

Low mass X-ray binaries (LMXBs) are binary systems that typically host a compact object, that can be either a neutron star (NS) or a stellar-mass black hole (BH), and a companion star, that is normally a main sequence low mass star $\left(M<M_{\odot}\right)$. The mechanism that causes the $\mathrm{X}$-ray emission is the accretion of matter coming from the companion star onto the compact object. This occurs through Roche lobe overflow, during which the companion star, filling its Roche lobe, loses mass in favour of the compact object across the Lagrangian point $L_{1}$ of the system, building an accretion disc.

Some of these sources are persistent, with a constant, high X-ray luminosity at a level of $10^{37}-10^{38} \mathrm{erg} \mathrm{s}^{-1}$; others are transient, i.e., possess a luminosity that varies between periods of enhanced activity (called outbursts), characterised by a duration of months and a luminosity that is comparable to that of persistent systems, and longer periods of quiescence, that typically last for several years, with a luminosity of $\sim 10^{30}-10^{33} \mathrm{erg} \mathrm{s}^{-1}$.

NIR-optical observations of LMXBs during quiescence offer the only chance to study the characteristics of the companion star. If the latter suffers significant tidal distortion, the light curves in quiescence are characterized by orbital variability due to ellipsoidal modulation. Otherwise, if strong irradiation effects are present (such as in case of tight systems), the light curve will be almost sinusoidally modulated at the system orbital period. 
During outburst instead, the main emitter at NIR-optical frequencies are typically the bright accretion disc outer regions. Moreover, in some cases the emission of partially self-absorbed, relativistic outflows (jets) can also be detected during outburst. Such jets are normally associated with hard X-ray spectral states, and seem to be quenched in correspondence to soft X-ray spectral states (accretion-ejection coupling paradigm, valid for BH LMXBs, yet to be confirmed for NS LMXBs; [1,2]). In the past few years, new evidence for jet emission in NS LMXBs have been reported. Despite the recent progresses in the study of black hole transients, only a partial picture has been built for NS X-ray binary jets, even if qualitative similarities with BH candidates have been found.

In the radio band the jets observed during the hard X-ray states have a flat or slightly inverted spectrum, due to the superposition of self-absorbed synchrotron spectra [3]. Above certain frequencies this flat spectral component should break to an optically thin spectrum corresponding to the point at which the entire jet becomes transparent. There is evidence both from some BH and NS X-ray binaries that this break occurs around the NIR spectral region (e.g., [4,5]). Thus the case is strong that there is a significant contribution of the jet in the NIR-optical regime of LMXBs. This contribution is typically detectable in the spectral energy distribution (SED) of the systems as an infrared excess with respect to the multi-colour black body of the accretion disc.

\section{Results}

\subsection{The Ultra-Compact X-ray Binary $4 U$ 0614+091}

The system $4 \mathrm{U} 0614+091$ is an ultra-compact X-ray binary (UCXB) candidate, i.e., a LMXB with short orbital period (typically $<80 \mathrm{~min}$ ), which causes the density of the companion star to be higher than the typical density of main sequence stars. In particular, the observation of the candidate optical spectrum [6,7] allowed to conclude that the system possesses a carbon-oxygen accretion disk, and that the companion star must be an oxygen-rich, evolved star. The orbital period of the system is unknown to date, despite the several attempts to measure it [7-11].

We observed 4U 0614+091 on 27 January 2013 using the PAOLO polarimeter mounted at the 3.6 m FGG TNG telescope at La Palma. The Stokes parameters of the linear polarisation (LP) are measured by rotating the instrument at four different angles with respect to the telescope axis (i.e., the position angles $\left.\Phi=0^{\circ}, 45^{\circ}, 90^{\circ}, 135^{\circ}\right)$. In total, we obtained a set of $28 r$-band $(6200 \AA)$ images of $240 \mathrm{~s}$ integration each. The night was clear, with seeing degrading with time (from $1.0^{\prime \prime}$ to $1.6^{\prime \prime}$ ). We carried out image reduction by subtracting an average bias frame and dividing by a normalized flat frame. We performed flux measurements for all the objects in the field of view through aperture photometry using daophot [12]. See [13] for details on the PAOLO polarimeter structure and usage.

With the aim to enhance the $\mathrm{S} / \mathrm{N}$ ratio, we averaged all the images with good seeing (we defined a limit to the seeing of $<1.3^{\prime \prime}$, which allowed us to use 12 images, corresponding to 48 min observation). We evaluated the Stokes parameters $Q$ and $U$ of all the objects in the field using the instrumental polarization model described in [13].

The field stars we chose as references clustered well around a common value in the $Q-U$ plane (Figure 1, left panel); this allowed us to assume them as intrinsecally unpolarised and to consider the interstellar polarisation of the field to be low. We could thus correct the values of $Q$ and $U$ of the target for the average $Q$ and $U$ obtained for the field stars, in order to account for the not negligible effects of instrumental polarisation. The value of the correction is reported in Table 1.

Considering these average Stokes parameters (Table 1), that can be supposed to be normally distributed, we used a Monte Carlo simulation to obtain the probability distribution that describes the polarisation degree $P$ of the radiation (Rice distribution, [14]; see [7] for a full description of the model). We then fit this distribution with a Gaussian function, and we evaluated the most probable value of the average $r$-band LP to be $P=2.85 \pm 0.96 \%$, that is significant at a $3 \sigma$ level. 

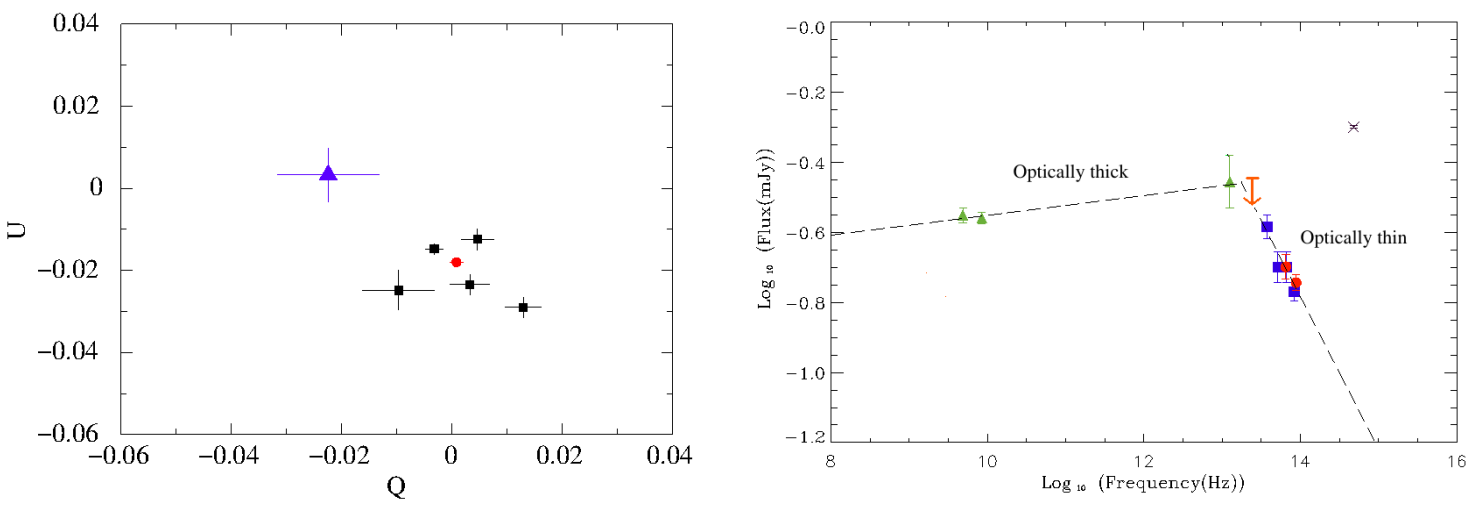

Figure 1. Left panel: $U$ vs. $Q$ for the averaged image of the optical $r$ filter, for $4 \mathrm{U} 0614+091$ (blue triangle) and for five reference field stars (black squares), Table 1 . With a red dot we indicated the weighted mean of the reference stars Stokes parameters. The parameters reported are not corrected for interstellar or instrumental effects. Right panel: Spectral energy distribution of the system $4 \mathrm{U}$ 0614+091 built starting from the radio (green triangles) and infrared points (blue squares) of [4], from the ALL WISE catalog magnitudes (red circles) and from the $r$-flux obtained in this work (black $x)$. With an orange arrow we indicated the W3-band WISE upper limit obtained from the ALLWise catalog. Superimposed, the two linear fits of the spectral energy distribution (SED). All the points are normalized to the fluxes of [4].

Table 1. Values of the $Q$ and $U$ Stokes parameters not corrected for interstellar or instrumental effects represented in Figure 1 (left panel) for 4U 0614+091 and for five reference field stars. The weighted mean of the reference stars Stokes parameters has been reported in the last row and corresponds to the amount of correction that we applied to $Q$ and $U$ of the target.

\begin{tabular}{ccc}
\hline Object & $Q \mathbf{~ ( \% )}$ & $\boldsymbol{U} \mathbf{( \% )}$ \\
\hline 4U 0614+091 & $-2.24 \pm 0.93$ & $0.32 \pm 0.66$ \\
\hline Star 1 & $-0.32 \pm 0.17$ & $-1.47 \pm 0.13$ \\
Star 2 & $0.46 \pm 0.29$ & $-1.24 \pm 0.26$ \\
Star 3 & $1.29 \pm 0.33$ & $-2.90 \pm 0.26$ \\
Star 4 & $-0.97 \pm 0.65$ & $-2.50 \pm 0.49$ \\
Star 5 & $0.32 \pm 0.36$ & $-2.35 \pm 0.26$ \\
Weighted mean & $0.08 \pm 0.12$ & $-1.80 \pm 0.10$ \\
\hline
\end{tabular}

\subsection{The Transitional Millisecond Pulsar PSR J1023+0038}

The "missing link" pulsar PSR J1023+0038 was the first LMXB that showed the potential to alternate between a radio pulsar phase, powered by rotation, and an X-ray state that is powered by accretion. This system was initially observed in the radio band [15], and later showed optical signs indicative of an accretion disc [16], which led to identify it as a NS LMXB. In 2007 then [17] detected a $1.69 \mathrm{~ms}$ radio pulsar as compact object of the system. After that, PSR J1023+0038 underwent a state change from the MSP state to an accretion-disc dominated state in June 2013 [18]. Because of the transitions that systems like PSR J1023+0038 undergo from an accretion state to a rotation powered state, they are often referred to as transitional MSPs. The system orbital period is $4.75 \mathrm{~h}$ [19], and the companion is a G-type star with mass of $0.2 \mathrm{M}_{\odot}$ [19]. The distance is $\sim 1.37 \mathrm{kpc}$ [20].

We observed PSR J1023+0038 on 8 February 2015 with the EFOSC2 instrument in polarimetric mode mounted on the ESO New Technology Telescope (NTT) in La Silla (Chile), using the optical BVR $i$ filters. The night was photometric, with seeing constant at a level of $\sim 0.6-0.8^{\prime \prime}$. We performed image reduction following the standard procedure of subtraction of an average bias frame and division by a 
normalised flat frame. We performed the flux measurements by using aperture photometry techniques on the fields of the two targets with daophot [12].

Differently from PAOLO, the EFOSC2 polarimeter mounts a rotating half wave plate (HWP), thanks to which we could obtain observations at four different angles with respect to the telescope axis without rotating the whole instrument. In particular, we considered the four HWP angle $\Psi=0^{\circ}, 22.5^{\circ}, 45^{\circ}, 67.5^{\circ}$, which correspond to a rotation of the sky of $0^{\circ}, 45^{\circ}, 90^{\circ}, 135^{\circ}$, respectively.

Alternating the filters, we obtained for the target a set of five 60s integration images, for each filter and HWP angle.

We first summed all the five images in each optical band together, to enhance the $S / N$, and we evaluated a parameter, $S(\Psi)$, for each HWP angle $\Psi$, starting from the ordinary $(o-)$ and extraordinary (e-) fluxes of the target, $f^{o}(\Psi)$ and $f^{e}(\Psi)$, in which the incident radiation is splitted by the polarimeter, and from the averaged ratio between $o$ - and $e$-fluxes of some unpolarised field stars $f_{\mathcal{u}}^{o}(\Psi)$ and $f_{\mathcal{u}}^{e}(\Psi)$. In particular, this parameter can be expressed as $([21,22])$ :

$$
S(\Psi)=\left(\frac{f^{o}(\Psi) / f^{e}(\Psi)}{\left\langle f_{\mathcal{u}}^{o}(\Psi) / f_{\mathcal{u}}^{e}(\Psi)\right\rangle}-1\right) /\left(\frac{f^{o}(\Psi) / f^{e}(\Psi)}{\left\langle f_{\mathcal{u}}^{o}(\Psi) / f_{\mathcal{u}}^{e}(\Psi)\right\rangle}+1\right)
$$

This parameter can be regarded as the component of the normalised Stokes vector that describes LP along the direction selected by the HWP angle $\Psi$. The relation between $S, P$, and the LP angle $\theta$ is given by:

$$
S(\Psi)=P \cos 2(\theta-\Psi) .
$$

The LP values resulting from the fit of the $S$ parameter with Equation (2) in each band are reported in Table 2.

\begin{tabular}{|c|c|c|c|}
\hline$B$ & $V$ & $R$ & $i$ \\
\hline \multicolumn{4}{|c|}{$\mathrm{P}(\%)$} \\
\hline $1.17 \pm 0.51$ & $1.09 \pm 0.27$ & $0.90 \pm 0.17$ & $0.55 \pm 0.22$ \\
\hline \multicolumn{4}{|c|}{$\mathrm{P}(3 \sigma$ upper limit) } \\
\hline $2.7 \%$ & - & - & $1.21 \%$ \\
\hline \multicolumn{4}{|c|}{ Interstellar/instrumental LP ( $3 \sigma$ upper limit) } \\
\hline $1.23 \%$ & $0.63 \%$ & $0.48 \%$ & $0.54 \%$ \\
\hline
\end{tabular}

Table 2. Results of the optical polarimetry performed on PSR J1023+0038.

We obtain significant $(>3 \sigma)$ detections of LP just in $V$ - and $R$-bands, the first being slightly higher than the latter. The $3 \sigma$ upper limits to the LP in the $B$ and $i$ bands are reported in Table 2. Interestingly, in the $i$-band we obtained a very constraining upper limit, which suggests a decreasing trend of the LP towards longer wavelengths.

Then we searched for possible phase-dependent variations of the LP in the $V$ and $R$ bands, by analyzing the five datasets for each band separately. The orbital phases have been obtained from the ephemeris reported in [19].

Differently from what happens in the $V$-band, the $R$-band polarization curve deviates from a constant, and its shape suggests a sinusoidal trend (Figure 2, left bottom panel). The fit with a sinusoidal function with period fixed to the orbital period of the target $(4.75 \mathrm{~h})$ gives a good $\chi^{2} /$ dof $(0.11 / 2)$. If we compare the $R$-band light curve with the polarisation curve in this band, the positions of the maxima of the two curves are both located around phase 0.5 (superior conjunction of the companion star, i.e., when the observer sees the irradiated face of the donor), the two positions being consistent with each other within $1 \sigma$ (maximum of the light curve phase: $0.46 \pm 0.01$; maximum of the polarisation curve phase: $0.57 \pm 0.13$; errors at the $68 \%$ confidence level). It must be noted that the fit of the $R$-band polarisation curve with a constant model is still preferable to the constant + sinusoidal one, despite the high $\chi^{2} /$ dof of the former (according to an $F$-test, the addition of a sinusoidal function 
gives a chance probability of $\sim 1.5 \times 10^{-2}$, corresponding only to a marginally $2.6 \sigma$ improvement in the fit). Nevertheless, an intriguing increase of the polarisation degree towards phase 0.5 is clearly observed (Figure 2, left panel).
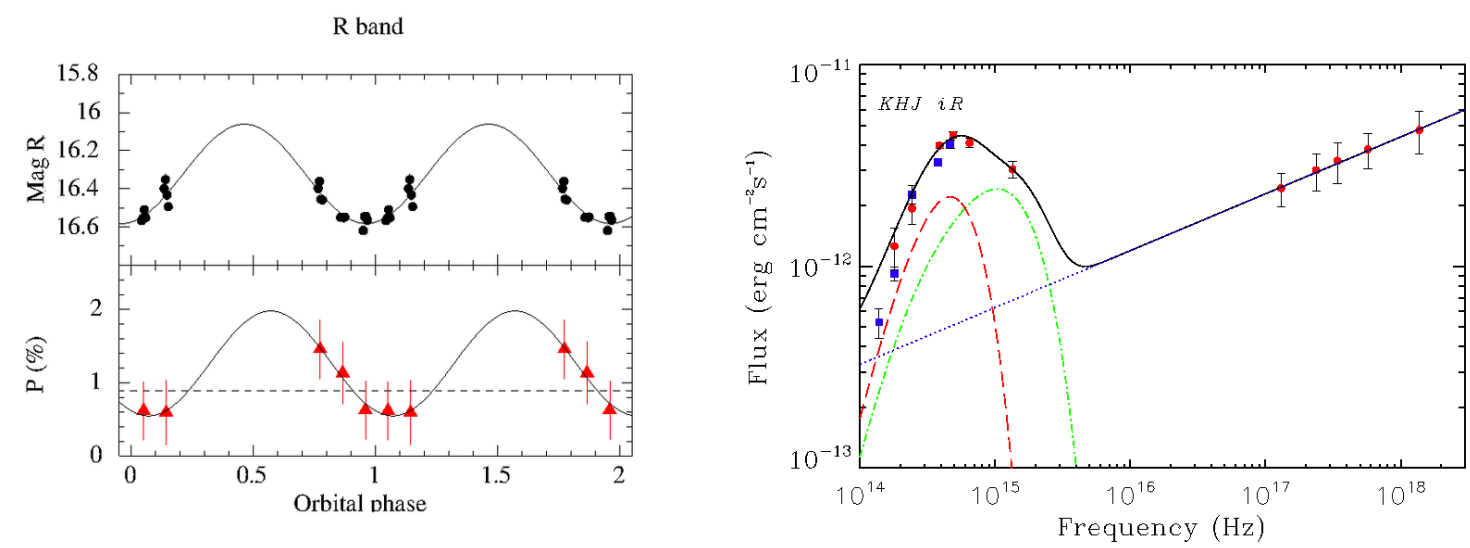

Figure 2. Left panel, up: $R$-band light curve of J1023 (magnitude vs. orbital phase). With a solid line, a fit of the light curve with a sinusoidal function with period fixed to 1 (corresponding to $4.75 \mathrm{hr}$ ) is reported. The $\chi^{2} /$ dof of the fit is $11.35 / 17$; bottom: $R$-band polarisation curve represented with respect to the orbital phase of the system. Superimposed, the fits with a constant function (dashed line; $\chi^{2} /$ dof $=3.66 / 4$ ) and with a sinusoidal function with period fixed to 1 (solid line; $\chi^{2} /$ dof $=0.11 / 2$ ). Errors are represented at the $68 \%$ confidence level. Two periods are drawn for clarity. Right panel: NIR- optical contemporaneous SED of J1023 obtained in this work (orbital phase 0.59; blue squares); the NIR-X-rays SED of J1023 reported in [23] is also represented (red dots), with superimposed the fit with a model made by the companion star (red dashed line), the accretion disc (green dash-dotted line) and the shock emission powered by the neutron star (NS) spin down luminosity (blue dotted line).

\section{Discussion}

The production of relativistic jets can be a possible origin of the LP in LMXBs. Jets in fact emit synchrotron radiation, that is intrinsically linearly polarised up to high percentages, if the magnetic field at the base of the jet is ordered.

For the majority of BH and NS LMXBs in which jets have been observed, the optical and NIR LP do not exceed a few per cent, meaning that the level of ordering of the magnetic fields at the base of the jets is usually low (e.g., [24,25]).

In the case of the UCXB 4U 0614+091, the emission of a jet was already stated in [4], thanks to the observation of an IR excess in the SED; we thus expected to confirm this evidence through the detection of a LP in the optical of a few per cent, under the hypothesi of tangled magnetic fields at the base of the jet. In our work, we measured a $3 \sigma$ significant average LP degree of $2.85 \% \pm 0.96 \%$ in the $r$-band, in agreement with our expectation. Such a low optical LP could also be ascribed to electron scattering (Thomson scattering) of radiation with free electrons, that are usually found in the ionized accretion discs of LMXBs. However, in order to have free electrons, hydrogen in the disc is required. As stated in [6] and [7], no hydrogen is present in the accretion disc of 4U 0614+091, thus allowing us to exclude this possibility. We could therefore safely interpret our LP measurment as due to synchrotron radiation emitted from a relativistic particles jet, thus confirming the results reported in [4].

We then built the SED of the system, following [4]. We added to the NIR and radio points reported there the archival data that we found in the ALLWISE catalog (orange arrow and red circles in Figure 1, right panel) and our $r$-band point. The fit with a linear function allowed us to obtain a spectral index $\alpha$ of $\sim 0.03$ in the optically thick spectrum, whereas in the optically thin part $\alpha \sim-0.43$, 
that is consistent with [4]. The jet-break frequency $v_{\text {break }}$ moreover is found in the same range of [4] $\left(1.25 \times 10^{13} \mathrm{~Hz}<v_{\text {break }}<3.71 \times 10^{13} \mathrm{rHz}\right)$.

Assuming that a jet has been launched, the linear fit to the NIR SED reported in Figure 1 (right panel) allowed us to estimate the expected $r$-band flux due to the jet only ( $F_{\text {jet }} \sim 0.1 \mathrm{mJy}$ ). However, we knew the total de-reddened $r$-band flux of $4 U$ 0614+091 at the time of our observations to be $F_{\mathrm{r}} \sim 0.5 \mathrm{mJy}$. From the ratio $F_{\text {jet }} / F_{\mathrm{r}}$, we could thus obtain an estimate of the intrinsic LP of the jet of $\sim 15 \%$.

Also in the case of the transitional MSP PSR J1023+0038 we detected a low but significant optical LP. Particularly intriguing was the observation of the possible oscillation of the $R$-band polarisation curve at the system orbital period ( $4.75 \mathrm{~h}$; Figure 2, left panel). The position of the LP maximum seems to coincide within the errors with that of the light curve, i.e., phase $\sim 0.5$ (i.e., superior conjunction-when the observer sees the irradiated face of the companion star).

Let us first suppose that the observed LP might be ascribed to electron scattering. When the donor star is at superor conjunction, the disc is found along the line of sight between the observer and the companion star; thus this is the phase in which the effect of Thomson scattering should be maximum, if the disc is sufficiently extended and thick to allow the radiation emitted from the companion star to cross it, and so to become linearly polarised due to electron scattering. On the contrary, when the star is at inferior conjunction (phase 0), the radiation emitted from the companion star is no longer intercepted by the accretion disc, which would imply a decrease in the LP of the total optical emitted light, as observed (Figure 2, left panel).

The X-ray luminosity of PSR J1023+0038 at the time of our observations is some orders of magnitude lower than the typical luminosities of NS LMXBs for which jets have been detected [26]. Nevertheless, [27] measured for this source a radio flux that is compatible with the emission of a jet; therefore, we could not in principle exclude that our LP measurment could be due to synchrotron emission from a relativistic particles jet characterized by strongly tangled magnetic fields, in order to explain our really low LP values. We thus built the SED of the system to search for evidence of jet emission through the observation of any non-thermal components in the spectrum (as it happened in the case of $4 \mathrm{U}$ 0614+091; see Figure 1, right panel). To do that, we started from the broadband SED of the system that our group published in [23], based on a 2013 dataset, and we added the photometry reported in this work. To obtain a simultaneous SED by combining the two datasets, we fixed the semi-amplitudes of the light curves in the two filters that are in common between the two works (i.e., the $R$ and $i$ bands) to the values reported in [23], since the photometric analysis in that paper was more refined and accurate, and we repeated the fit of our light curves, under the hypothesis that the source did not vary considerably between 2013 and 2015. In this way, we could obtain the $i$ - and $R$-band fluxes at the same orbital phase as the NIR observations (we chose phase 0.59 , that is the orbital phase at which the $J$-band data have been collected). The obtained SED is shown in Figure 2, right panel.

The NIR-optical SED obtained in this work is well described by the best fit of the 2013 dataset reported in Figure 2, right panel. In particular, the 2013 dataset $J-H$ colour $(0.34 \pm 0.31)$ is consistent with the one derived in 2015 (0.22 \pm 0.05$)$, suggesting that the components involved in the 2013 NIR emission, i.e., the irradiated companion star and the accretion disc, remained unchanged in 2015. Therefore, NIR polarisation from a relativistic particles jet at the time of our polarimetric observations can be safely excluded.

We conclude that the optical LP of PSR J1023+0038 is more probably due to Thomson scattering with the electrons in the accretion disc rather than to synchrotron emission from a jet, since no evidence for the presence of such phenomenon has been found in our optical and NIR photometry.

The results presented here are are based on $[7,28]$.

Acknowledgments: M.C.B. thanks E. Angelakis and the organizers of the conference "Polarized emission from Astrophysical Jet" (Ierapetra, 12-16 June 2017) for accepting her contribution in the form of an oral presentation. 
Author Contributions: All authors contributed to the analysis reported in the paper.

Conflicts of Interest: The authors declare no conflict of interest.

\section{References}

1. Russell, D.M.; Miller-Jones, J.C.A.; Maccarone, T.J.; Yang, Y.J.; Fender, R.P.; Lewis, F. Testing the Jet Quenching Paradigm with an Ultradeep Observation of a Steadily Soft State Black Hole. Astrophys. J. Lett. 2011, 739, L19.

2. Muñoz-Darias, T.; Fender, R.P.; Motta, S.E.; Belloni, T.M. Black hole-like hysteresis and accretion states in neutron star low-mass X-ray binaries. Mon. Not. R. Astron. Soc. 2014, 443, 3270-3283.

3. Fender, R.P. Powerful jets from black hole X-ray binaries in low/hard X-ray states. Mon. Not. R. Astron. Soc. 2001, 322, 31-42.

4. Migliari, S.; Migliari1, S.; Tomsick, J.A.; Miller-Jones, J.C.A.; Heinz, S.; Hynes, R.I.; Fender, R.P.; Gallo, E.; Jonker, P.G.; Maccarone, T.J. The Complete Spectrum of the Neutron Star X-ray Binary 4U 0614+091. Astrophys. J. 2010, 710, 117-124.

5. Gandhi, P.; Gandhi, P.; Blain, A.W.; Russell, D.M.; Casella, P.; Malzac, J.; Corbel, S.; D'Avanzo, P.; Lewis, F.W.; Markoff, S.; et al. A Variable Mid-infrared Synchrotron Break Associated with the Compact Jet in GX 339-4. Astrophys. J. Lett. 2011, 740, L13.

6. Nelemans, G.; Jonker, P.G.; Steeghs, D. Optical spectroscopy of (candidate) ultracompact X-ray binaries: Constraints on the composition of the donor stars. Mon. Not. R. Astron. Soc. 2006, 370, 255-262.

7. Baglio, M.C.; Mainetti, D.; D’Avanzo, P.; Campana, S.; Covino, S.; Russell, D.M.; Shahbaz, T. Polarimetric and spectroscopic optical observations of the ultra-compact X-ray binary 4U 0614+091. Astron. Astrophys. 2014, 572, A99.

8. O'Brien, K. A Life with Stars. In Proceedings of the Honor of Ed van den Heuvel, Amsterdam, The Netherlands, 22-26 August 2005.

9. Zhang, Y.; Hynes, R.I.; Robinson, E.L. Millihertz quasi-periodic optical oscillations in 4U $0614+091$. Mon. Not. R. Astron. Soc. 2012, 419, 2943-2948

10. Shahbaz, T.; Watson, C.A.; Zurita, C.; Villaver, E.; Hernandez-Peralta, H. Time-Resolved Optical Photometry of the Ultracompact Binary 4 U 0614+091. Publ. Astron. Soc. Pac. 2008, 120, 848-851

11. Madej, O.K.; Jonker, P.G.; Groot, P.J.; van Haaften, L.M.; Nelemans, G.; Maccarone, T.J. Time-resolved X-Shooter spectra and RXTE light curves of the ultra-compact X-ray binary candidate 4U 0614+091. Mon. Not. R. Astron. Soc. 2013, 429, 2986-2996.

12. Stetson, P.B. DAOPHOT-A computer program for crowded-field stellar photometry. Publ. Astron. Soc. Pac. 1987, 99, 191-222.

13. Covino, S.; Molinari, E.; Bruno, P.; Cecconi, M.; Conconi, P.; D’Avanzo, P.; di Fabrizio, L.; Fugazza, D.; Giarrusso, M.; Giro, E.; et al. PAOLO: A Polarimeter Add-On for the LRS Optics at a Nasmyth focus of the TNG. Astrono. Nachr. 2014, 335, 117-123.

14. Wardle, J.F.C.; Kronberg, P.P. The linear polarization of quasi-stellar radio sources at 3.71 and 11.1 centimeters. Astrophys. J. 1974, 194, 249-255.

15. Bond, H.E.; White, R.L.; Becker, R.H.; Sean O'Brien, M. FIRST J102347.6+003841: The First Radio-selected Cataclysmic Variable. Publ. Astron. Soc. Pac. 2002, 114, 1359-1363.

16. Szkody, P.; Fraser, O.; Silvestri, N.; Henden, A.; Anderson, S.F.; Frith, J.; Lawton, B.; Owens, E.; Raymond, S.; Schmidt, G.; et al. Cataclysmic Variables from the Sloan Digital Sky Survey. II. The Second Year. Astron. J. 2003, 126, 1499-1514.

17. Archibald, A.M.; Kaspi, V.M.; Hessels, J.W.T.; Stappers, B.; Janssen, G.; Lyne, A. Long-Term Radio Timing Observations of the Transition Millisecond Pulsar PSR J1023+0038. ArXiv 2013, arXiv:astro-ph.HE/1311.5161.

18. Stappers, B.W.; Archibald, A.M.; Hessels, J.W.T.; Bassa, C.G.; Bogdanov, S.; Janssen, G.H.; Kaspi, V.M.; Lyne, A.G.; Patruno, A.; Tendulkar, S.; et al. A State Change in the Missing Link Binary Pulsar System PSR J1023+0038. Astrophys. J. 2014, 790, 39-47.

19. Archibald, A.M.; Stairs, I.H.; Ransom, S.M.; Kaspi, V.M.; Kondratiev, V.I.; Lorimer, D.R.; McLaughlin, M.A.; Boyles, J.; Hessels, J.W.T.; Lynch, R.; et al. A Radio Pulsar/X-ray Binary Link. Science 2009, 324, 1411-1414.

20. Deller, A.T.; Archibald, A.M.; Brisken, W.F.; Chatterjee, S.; Janssen, G.H.; Kaspi, V.M.; Lorimer, D.; Lyne, A.G.; McLaughlin, M.A.; Ransom, S.; et al. A Parallax Distance and Mass Estimate for the Transitional Millisecond Pulsar System J1023+0038. Astrophys. J. Lett. 2012, 756, L25. 
21. Di Serego Alighieri, S. Instrumentation for Large Telescopes; Cambrigre University Press: Cambrige, UK, 1998; Volume 199, p. 287.

22. Covino, S.; Lazzati, D.; Ghisellini, G.; Saracco, P.; Campana, S.; Chincarini, G.; di Serego, S.; Cimatti, A.; Vanzi, L.; Pasquini, L.; et al. GRB 990510: Linearly polarized radiation from a fireball. Astron. Astrophys. 1999, 348, L1-L4.

23. Coti Zelati, F.; Baglio, M.C.; Campana, S.; D’Avanzo, P.; Goldoni, P.; Masetti, N.; Muñoz-Darias, T.; Covino, S.; Fender, R.P.; Jiménez Bailón, E.; et al. Engulfing a radio pulsar: The case of PSR J1023+0038. Mon. Not. R. Astron. Soc. 2014, 444, 1783-1792.

24. Schultz, J.; Hakala, P.; Huovelin, J. Polarimetric Survey of Low-Mass X-ray Binaries. Balt. Astron. 2004, 13, 581-595.

25. Russell, D.M.; Casella, P.; Fender, R.; Soleri, P.; Pretorius, M.L.; Lewis, F.; van der Klis, M. Rapid variations of polarization in low-mass X-ray binaries. ArXiv 2011, arXiv:astro-ph.HE/1104.0837.

26. Campana, S.; Coti Zelati, F.; Papitto, A.; Rea, N.; Torres, D.F.; Baglio, M.C.; D’Avanzo, P. A physical scenario for the high and low X-ray luminosity states in the transitional pulsar PSR J1023+0038. Astron. Astrophys. 2016, 594, A31.

27. Deller, A.; Degenaar, N.; Hessels, J.; Heinke, C.; Jaodand, A. Radio detection of the transient neutron star LMXB 1RXS J180408.9-342058. The Astronomer's Telegram, 24 December 2015.

28. Baglio, M.C.; D’Avanzo, P.; Campana, S.; Coti Zelati, F.; Covino, S.; Russell, D.M. Different twins in the millisecond pulsar recycling scenario: Optical polarimetry of PSR J1023+0038 and XSS J12270-4859. Astron. Astrophys. 2016, 591, A101.

(C) 2017 by the authors. Licensee MDPI, Basel, Switzerland. This article is an open access article distributed under the terms and conditions of the Creative Commons Attribution (CC BY) license (http:/ / creativecommons.org/licenses/by/4.0/). 